\title{
THE USE OF THE MITOCHONDRIAL TRANSMEMBRANE ELECTRIC POTENTIAL AS AN EFFECTIVE BIOSENSOR IN ECOTOXICOLOGICAL RESEARCH
}

\author{
Eduardo M. da Silva ${ }^{\mathrm{a}^{1} 2}$, Amadeu M.V.M. Soares ${ }^{\mathrm{b}}$ and António J.M. Moreno ${ }^{\mathrm{c}}$ \\ a Instituto de Biologia da UFBa, Campus de Ondina, 40210-170 Salvador, BA, Brazil \\ ${ }^{\mathrm{b}}$ Instituto do Ambiente e Vida, Universidade de Coimbra, 3000 Coimbra, Portugal \\ ${ }^{\mathrm{c}}$ Centro de Neurociências de Coimbra, Universidade de Coimbra, 3000 Coimbra, Portugal
}

(Received in Germany 14 August 1997; accepted 11 November 1997)

\begin{abstract}
In this work, the mitochondrial transmembrane electric potential $(\Delta \Psi)$ of isolated mitochondria was used to evaluate the toxicity of some chemicals (endosulfan, 3,4-dichloroaniline, parathion, tributyltin and cadmium) and wastewater. Mitochondria were isolated from rat liver, and the $\Delta \Psi$ measured in a suitable assay medium, using a sensitive tetraphenylphosphonium (TPP+ ${ }^{+}$electrode. The test substance was preincubated in a rotenone-containing medium during $3 \mathrm{~min}$ with $1.0 \mathrm{mg}$ of mitochondrial protein. Mitochondria were energised with succinate and after the establishment of a constant maximal potential ADP was added to induce the phosphorylative cycle. Chosen endpoints were the membrane potential from mitochondria oxidising succinate and the depolarisation induced by ADP. After the appropriate transformations the EC50 (effective concentration) was calculated for each toxicant. Even very low concentrations of a toxicant were able to affect the $\Delta \Psi$, thus showing its suitability as a biosensor in ecotoxicology and results were reproducible between tests. The utilisation of $\Delta \Psi$ in screening tests of pure substances and wastewater seems to be very effective and can be carried out routinely.

C1998 Elsevier Science Ltd. All rights reserved
\end{abstract}

1 To whom correspondence should be addressed.

2 Present address: Instituto do Ambiente, Departamento de Zoologia, Universidade de Coimbra, 3000 Coimbra, Portugal. 


\section{Introduction}

Alterations in biochemical systems are often more sensitive indicators than those at higher level of biological organisation [1]. Over the past decade, scientists have drawn attention to this question searching for biochemical, physiological, and histological changes caused by exposure to pollutants. The mitochondria are a potential cellular target, as they are responsible for numerous vital reactions, playing a crucial role of biological significance as ATP producers.

Most of the ATP used in endergonic reactions is produced in the mitochondria via oxidative phosphorylation. Eukaryotic cells and aerobic bacteria present a high level of similarity in structure and function of mitochondria. Additionally, they can be isolated and remain viable (coupled) for some time. Moreover, mitochondria may be good indicators of systemic toxicity; in that a toxic response in form of inhibited mitochondrial functioning has a profound effect on cellular metabolism and may lead to cell death [2]. These features together allow the use of this organelle as an adequate biosensor in ecotoxicological research.

During the last 30 years, there were considerable advances in the use of isolated mitochondria to estimate the effects of chemical compounds on the oxidative phosphorylation in mammals, fish, plants, and in some micro-organisms [3-8], as the impairment of oxidative phosphorylation can be understood as a toxic effect to the cells. More recently, it was demonstrated that the transmembrane electric potential of mitochondria $(\Delta \Psi)$ can be altered when exposed to some toxicants, as such: DDT, 2,4-D, dinoseb, paraquat and cationic detergents [9-12]. However, these studies did not generate quantitative information, that enable the establishment of ecotoxicological indices, such as $\mathrm{EC}_{50}$ (effective concentration that causes $50 \%$ inhibition of a biological/biochemical process, in the specific case, oxidative phosphorylation). Indeed, the utilisation of the $\Delta \Psi$ has not been addressed yet in ecotoxicological research, although this is a vital force in bioenergetics.

In this work we designed a series of experiments to supply information on the effects of two organochlorine compounds: endosulfan $(6,7,8,9,10,10$-hexachoro-1,5,5a,6,9,9a-hexahydro-6,9-methano2.4.3-benzo-dioxathiepin-3-oxide) and 3,4-dichloroaniline (3,4-dichlorobenzeneamine); an organophosphate compound, parathion (O,O-diethyl O-p-nitrophenyl phosphorothioate); an organotin compound, tributyltin (TBT); a substance of reference, cadmium (cadmium chloride); and treated and non-treated domestic wastewater from the city of Coimbra (Portugal), on the $\Delta \Psi$ of isolated mitochondria. Data were statistically transformed accordingly to allow the calculation of EC50 (effective concentration) values. 
Materials and Methods

Milli-Q water was always used as dilution water. Succinate, ADP, rotenone, mannitol, HEPES, EGTA, fatty acid free bovine albumin, FCCP and cadmium chloride $(99.6 \%$ purity) were from SIGMA. Endosulfan ( $99.1 \%$ purity), parathion (98.9\% purity) and 3,4-dichloroaniline (3,4-DCA) (99.0\% purity) were acquired from Dr. Ehrenstorfer (Germany), and tributyltin chloride ( $96 \%$ of purity) from MERCK. All other reagents were of analytical grade.

Liver mitochondria were obtained from male Wistar rats $(200-300 \mathrm{~g})$ grown under standardised conditions, utilising a slightly modified version of the technique described by Moreno [13]. Animals were cervical stunned and decapitated. Liver tissues in pieces were rapidly excised, blotted, minced and washed several times in homogenisation buffer ( $225 \mathrm{mM}$ mannitol, $75 \mathrm{mM}$ sucrose, $10 \mathrm{mM}$ HEPES, 1.0 mM EGTA, 0.1\% defatted bovine serum albumin, $\mathrm{pH}$ 7.4). The preparation was homogenised using a Potter-Elvehjem homogeniser $(300 \mathrm{rpm}, \sim 5$ strokes) at a ratio of approximately $1.0 \mathrm{~g}$ of tissue to 8 volumes of homogenisation buffer. These previous procedures and the centrifugation were carried out under low temperature $\left(2-4^{\circ} \mathrm{C}\right)$. The suspension was centrifuged at $750-\mathrm{x} g$ for $10 \mathrm{~min}$, and the resulting supernatant was transferred to another tube, discarding the remaining pellet. The tube was completed with homogenisation buffer and centrifuged at $12000-\mathrm{x} g$ for $10 \mathrm{~min}$. The brown mitochondrial pellet was gently resuspended in washing buffer, which had the same composition as the homogenisation buffer but EGTA and serum albumin ( $\mathrm{pH}$ 7.2), with the help of a fine painters' brush, after the suctioning of the supernatant and loose mitochondria. This procedure was repeated twice, transferring the resuspended media to a clean centrifuge tube. Finally, the pellet was resuspended in the same way, with approximately $2.0-\mathrm{ml}$ of the washing buffer. Mitochondrial protcin concentrations were measured according to the biuret method [14]. All substances were diluted in ethanol, with the exception of the water soluble cadmium chloride. Wastewater fractions were directly given to the assayed media without any carrier.

The mitochondrial transmembrane potential was estimated by calculating the transmembrane distribution of tetraphenylphosphonium (TPP ${ }^{+}$) with a $\mathrm{TPP}^{+}$-selective electrode [15]. This electrode was constructed with a polyvinylchloride-based membrane, containing tetraphenylboron as an ionexchanger. The membrane was glued to a polyvinyl chloride tube $(2 \mathrm{~mm}$ diameter and $\pm 75 \mathrm{~mm}$ length) with tetrahydrofuran. As an internal reference a $10 \mathrm{mM} \mathrm{TPP}^{+}$solution was placed inside the tube, in which a silver wire coated with $\mathrm{AgCl}$ made the connection with a suitable $\mathrm{pH}$-electrometer (Radiometer, Model PHM 84). The electromotive force between the TPP + electrode and an external reference $\mathrm{Ag} / \mathrm{AgCl}_{2}$ electrode in the sample solution was measured by the electrometer connected to a pen-writing recorder (Kipp and Zonen, model BD112). The voltage response of the electrode to log $\left[\mathrm{TPP}^{+}\right]$was linear, with a slope of $59 \pm 1$, in good agreement with the Nernst equation. In practice, the 
$\mathrm{TPP}^{+}$-electrode checking was routinely done by successive additions of a known concentration of $\mathrm{TPP}^{+}$, doubling each time the preceding concentration. Only electrodes with a maximum response $(\Delta \mathrm{E}=17,8 \mathrm{mV}$ ) were used. The $\Delta \Psi$ was measured in an open vessel containing $1.0 \mathrm{ml}$ of the assay medium (130 mM sucrose, $50 \mathrm{mM} \mathrm{KCl}, 5 \mathrm{mM} \mathrm{MgCl} 2,5 \mathrm{mM} \mathrm{KH} \mathrm{KOO}_{4}, 5 \mathrm{mM}$ HEPES, $2 \mu \mathrm{M}$ rotenone and $3 \mu \mathrm{M} \mathrm{TPP}+, \mathrm{pH} 7.4$ ), maintained at $25{ }^{\circ} \mathrm{C}$, under efficient magnetic stirring by continuously monitoring the movements of the TPP + across the mitochondrial membrane (Fig. 1). This low $\mathrm{TPP}^{+}$concentration was chosen to achieve high sensitivity in measurement and to avoid possible toxic effects on mitochondria [16-17]. Calibrations were also run in the presence of the tested toxicant to check for possible interferences.

The fluctuationns of $\Delta \Psi$ associated to succinate energisation and phosphorylative cycle upon ADP addition were estimated from the following equation, at $25^{\circ} \mathrm{C}$ :

$$
\Delta \psi(\mathrm{m} V)=59 * \log \left(\frac{v}{V}\right)-59 * \log \left(10^{\frac{\Delta E}{59}}-1\right)
$$

where $\mathrm{v}, \mathrm{V}$ and $\Delta \mathrm{E}$ are the volumes of mitochondria and incubation medium, and the deflection of the electrode potential from the baseline, respectively. A matrix volume of $1.1 \mu \mathrm{l} / \mathrm{mg}$ protein was assumed [18].

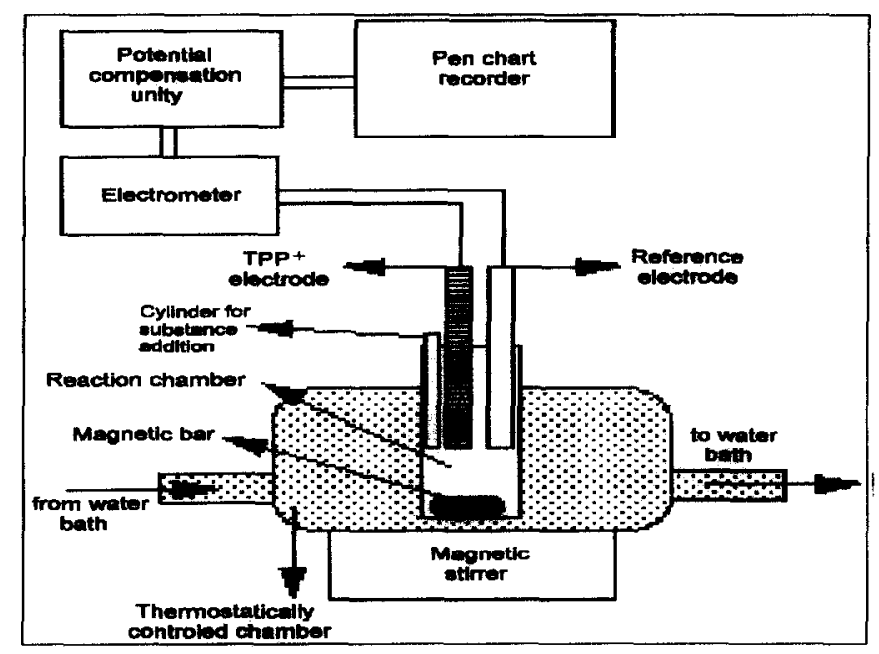

Fig. 1 - Schematic view of the TPP+ electrode.

No correction was made for the "passive binding" of TPP+ to the mitochondrial membranes, since the purpose of the experiments was to show relative changes in potentials rather than absolute values. Effects of toxicants on passive TPP+ binding measured under uncoupling conditions provoked by the addition of FCCP (carbonylcyanidep(trifluoromethoxy)phenylhydrazone) were also negligible. 
For each assay, different volumes of the test substance were added to a $1.0 \mathrm{mg}$ of mitochondrial protein, allowed to incubate for $3 \mathrm{~min}$ and then, energised with $5 \mathrm{mM}$ succinate. Upon addition of succinate, mitochondria immediately developed a membrane potential typical of state 4 . The oxidative phosphorylation cycle was initiated by the addition of a known amount of ADP after 2 to 4 min. The tightly coupled nature of mitochondria was tested in every assay, taking as the level of response and stability of the succinate develuped potential. According to preliminary studies, the chosen endpoints were: a) the capability of energisation of the mitochondria, measured as electric potential, after the succinate addition, for endosulfan, parathion, 3,4-DCA and cadmium, b) the depolarisation induced by ADP for the TBT and wastewater. These endpoints were chosen according to the different specific actions of the substances on mitochondrial metabolism. For instance, in the case of TBT and untreated wastewater, preliminary experiments showed that no detectable effects relatively to the control were noticed in the potential developed upon succinate energisation. This means that TBT and untreated wastewater neither affect the respiratory chain nor produce any uncoupling effect. Therefore, the lowering of the depolarisation induced by ADP was chosen as an endpoint for TBT and untreated wastewater, as it reflects specific actions on the mitochondrial phosphorylation system. Appropriate blanks and calibration tests were run in the presence of the test substance and ethanol in order to exclude any direct interference of the compound and its solvent in the results. Experiments were carried out testing different substance concentrations. The concentration responsible for no depolarisation after ADP addition (lack of phosphorylation) was taken as $100 \%$ effect and the succinate transmembrane electric potential in the absence of a given substance was considered as the $0 \%$ effect. However, in the case of TBT and domestic wastewater, the depolarisation induced by ADP in the control was considered as the $0 \%$ effect, since no detectable differences were noticed on the substrate energisation. Normalisation of data was achieved by employing the formula:

$$
\Delta \psi_{1}=\frac{\left(\Delta \psi \mathrm{C}_{\text {control }}-\Delta \psi \mathrm{Np}\right)}{(\Delta \psi n-\Delta \psi \mathrm{Np})}
$$

where $\Delta \Psi_{1}$ is the effect number index, $\Delta \Psi \mathrm{C}_{\text {control }}$ is the control value obtained with the addition of an amount of ethanol with no visible effect on the potential development, $\Delta \Psi \mathrm{Np}$ is the no-phosphorylation point given by the succinate generated electric potential, when no phosphorylation was registered, and $\Delta \Psi n$ is any point to be calculated. The predicted toxicant concentration to reduce the succinate generated potential or the depolarisation caused by ADP addition by $50 \%$ relative to controls, i.e. EC 50, was calculated using the inverse prediction by regressing electric potential against the square root of the toxicant final concentration, as applicable for Poisson distribution data [19]. 


\section{Results}

Isolated mitochondria, free of toxic compounds immediately develop a normal transmembrane potential upon addition of succinate between 210 and $220 \mathrm{mV}$ (negative inside). Addition of ADP, which induces transition to state 3, causes an immediate decrease of $\Delta \Psi$, since ATP-synthase uses $\Delta \Psi$ to phosphorylate added ADP. When the state 3 respiration cycle is completed, the transmembrane potential returns nearly to its initial value.

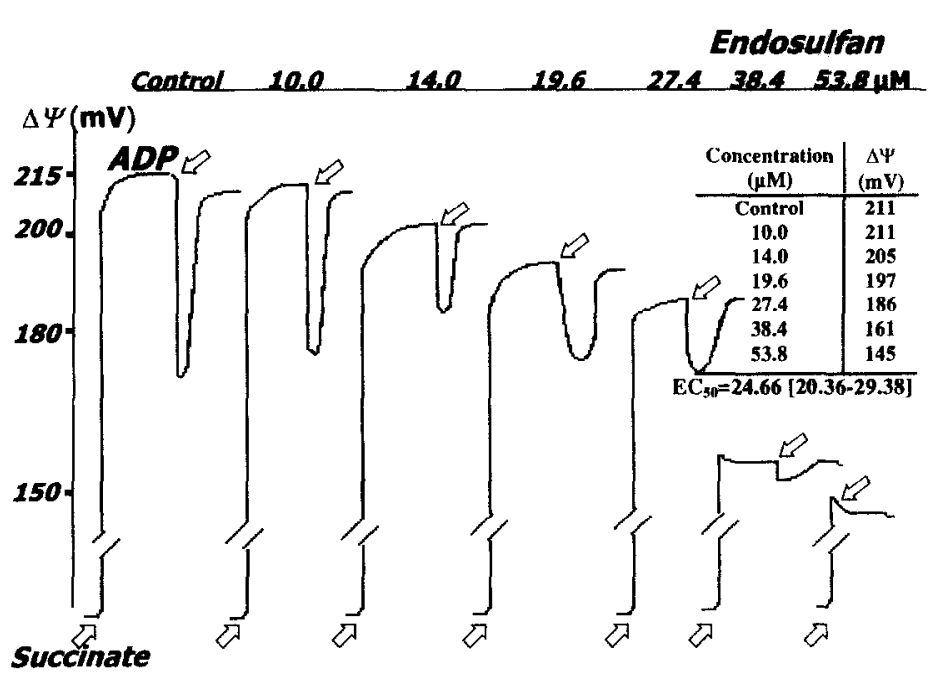

Fig. 2 - Effects of endosulfan on the mitochondrial $\Delta \Psi$.

In the presence of increasing concentrations of endosulfan, mitochondria consistently decrease the respiratory $\Delta \Psi$, when compared to the control. Additionally, mitochondria suffered a progressively lowering in depolarisation induced by ADP addition (Fig. 2). Moreover, increasing concentrations of this substance consistently decreased the depolarisation amplitude following ADP addition and delayed slightly the lag phase preceding repolarisation. At the highest concentration tested $(53.8 \mu \mathrm{M})$, the concentration of endosulfan to which no apparent depolarisation occurs, the succinate polarisation was $145 \mathrm{mV}$, against a control value of $-211 \mathrm{mV}$.

Even low concentrations of parathion induced mitochondria to develop a lower succinate polarisation, lower ADP depolarisation, lower recovery (return to initial polarisation state) and longer recovery time, when compared to controls (Fig. 3), confirming the results of Moreno [13]. It is interesting to note that by concentrations higher than $1.93 \mathrm{mM}$ there was a considerable delay in the lag-phase preceding 


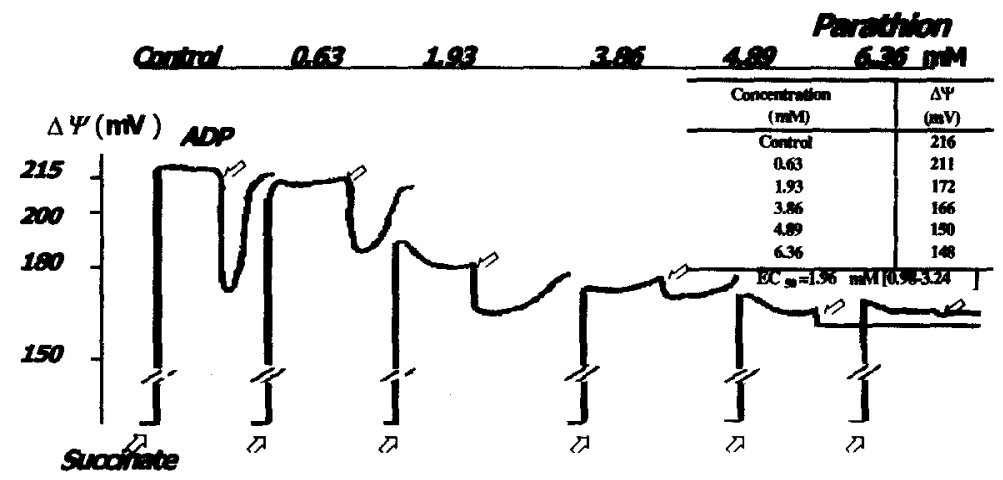

Fig. 3 - Effects of parathion on the mitochondrial $\Delta \Psi$.

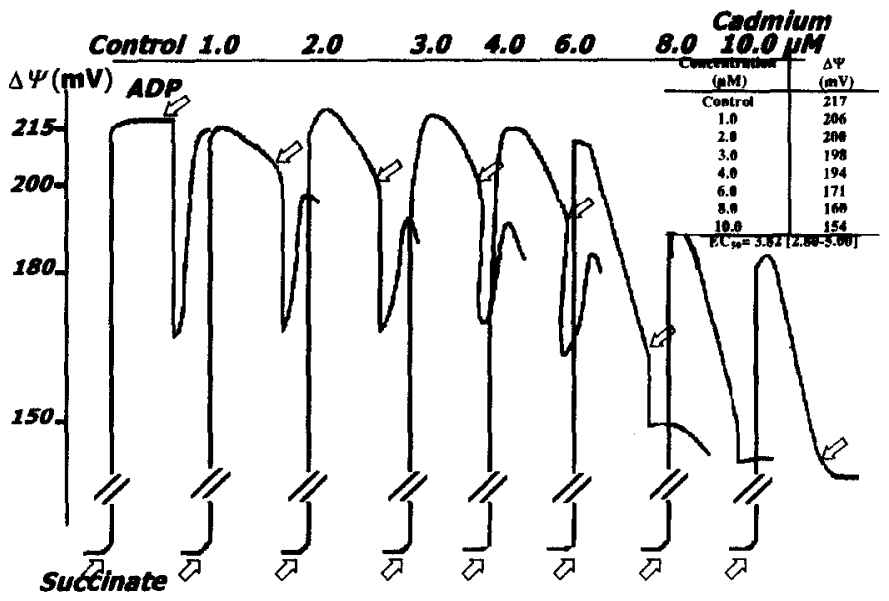

Fig. 4 - Effects of cadmium on the mitochondrial $\Delta \Psi$.

repolarisation. For this concentration, for instance, the succinate polarisation $(\Delta \Psi)$ was -172 , while the control reached $-216 \mathrm{mV}$. Similarly to endosulfan, increasing concentrations of this toxicant lead to a decrease in the succinate induced $\Delta \Psi$, accompanied also by a decrease of the depolarisation caused by ADP addition. 
As opposed to the others tested toxicants, cadmium incubated mitochondria showed a different pattern of effects. Even increasing concentrations of cadmium, up to $6.0 \mu \mathrm{M}$, seemed not to disrupt the initial level of succinate energisation (Fig. 4). However, notoriously the $\Delta \Psi$ is rapidly time-collapsed, even at the lowest concentration tested $(1.0 \mu \mathrm{M})$.

Mitochondria exposed to 3,4-DCA decreased the level of succinate energisation and depolarisation

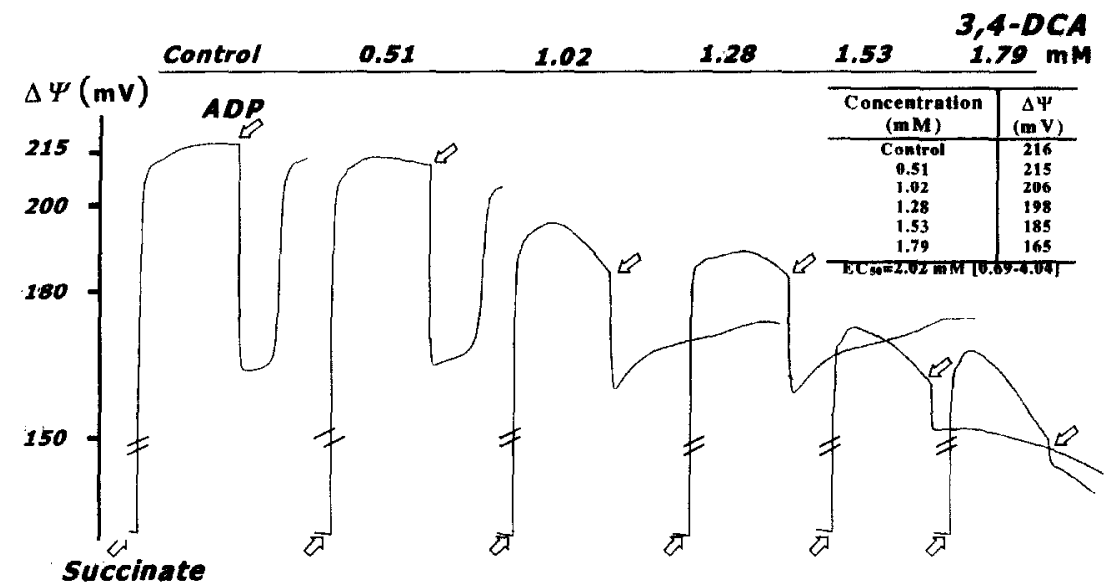

Fig. 5- Effets of 3,4-DCA on the mitochondrial $\Delta \Psi$.

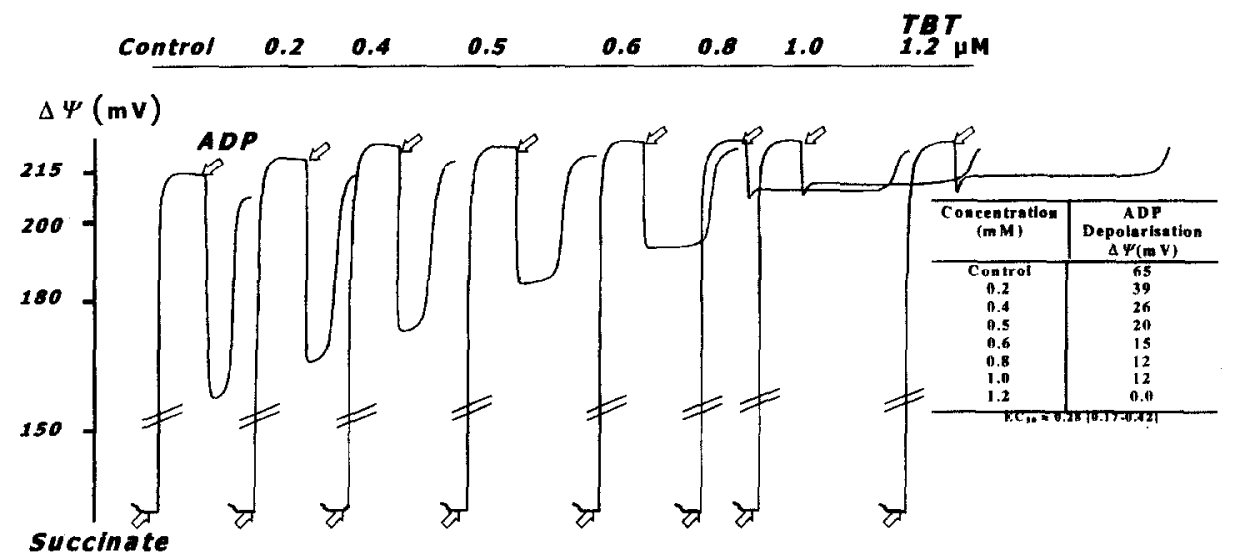

Fig. 6- Effects of TBT on the mitochondrial $\Delta \Psi$.

induced by ADP and also the $\Delta \Psi$ of succinate energisation did not hold and collapses gradually (Fig. 5), similarly to cadmium. Quantitatively speaking, cadmium was more toxic than 3,4-DCA, but qualitatively showed an almost identical pattern of concentration-response relationships than 3,4-DCA. 
TBT caused profound effects on the $\triangle \Psi A D P$ induced depolarisation (Fig. 6), without affecting the level of succinate energisation. In this particular case, by increasing concentrations of TBT, there is even a

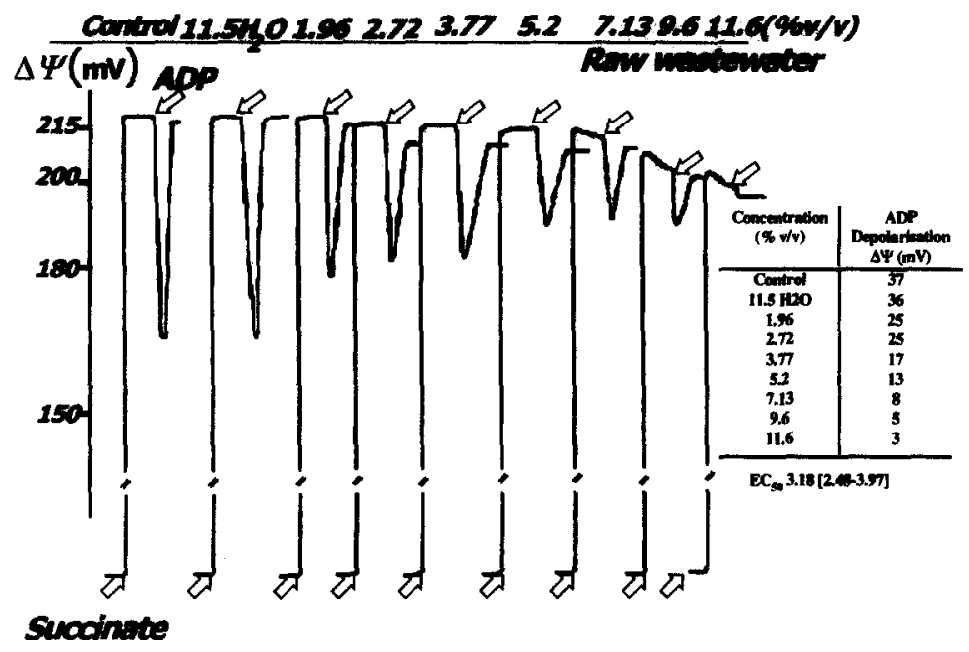

Fig. 7- Effects of raw wastewater on the mitochondrial $\Delta \Psi$.

slight increase of the $\Delta \Psi$ after the succinate addition.

Four wastewater fractions were tested from $24 \mathrm{hr}$ composed samples. The treated wastewater (effluent) did not show any toxicity even at the highest dilution tested $(11.5 \% \mathrm{v} / \mathrm{v})$. Similar results were found for the effluents leaving the primary clarifier, before entering the trickling filter and the clarifier, after it. On the other hand, raw wastewater (influent) induced the ADP $\Delta \Psi$ negatively, without no effects on the $\Delta \Psi$ after succinate addition (Fig. 7).

\section{Discussion}

Energy for mitochondrial ATP synthesis is provided by the proton electrochemical gradient generated by mitochondrial respiration according to the chemiosmotic hypothesis [20]. This electrochemical gradient of protons, usually called as protonmotive force $(\Delta p)$ is composed of two interconvertible components: the $\Delta \mathrm{pH}$ gradient $(\Delta \mathrm{pH}$, alkaline inside) and the transmembrane electric potential $(\Delta \Psi)$. The $\Delta \Psi$ determination is of major importance in studies of mitochondrial oxidative phosphorylation, hence it represents the main component of the $\Delta \mathrm{p}$, accounting for $90 \%$ of the total available energy [21]. The TPP + electrode allows an easy and precise means for the measurement of the $\Delta \Psi[9,15]$. 
The consisting decrease of $\Delta \Psi$ with increasing concentrations of endosulfan supports the idea that this substance is a general inhibitor of the electron transport chain of rat liver mitochondria [5]. The decrease of the phosphorylation efficiency may result not only from direct action on the redox chain but also from an enhanced permeabilisation to protons of the mitochondrial inner membrane, as demonstrated by Simões and Castanheira [22]. These authors applied the approach developed by Brand [23], consisting in the titration of non-phosphorylating mitochondria with a respiratory inhibitor and the simultaneous monitoring of the respiratory rates and the $\Delta \Psi$.

In the case of parathion, where the proportional increase in concentration did not produce a proportional visible effect, to reach a $100 \%$ effect (situation where there is no further ADP phosphorylation) a relatively high concentration was given. Therefore, $\mathrm{EC}_{50}$ results are biased due to this parathion feature. On the other hand, Yamano \& Morita [24] testing rat, and Wenzel et al [25] pig liver mitochondria oxygen consumption found no inhibition or uncoupling effect on mitochondria for dimethyl-parathion, an analogue of the diethyl-parathion. It seems parathion acts as a direct inhibitor of the respiratory chain, rather than showing uncoupling effects.

The collapsing of the $\Delta \Psi$ resultant from the succinate energisation caused by cadmium can be partly related to the inhibitory action on ubiquinone-b-citochrome cl complex [26]. However, the decrease in the phosphorylation efficiency caused by cadmium is mostly due to its strong uncoupler action of the oxidative phosphorylation [27]. This uncoupling action results from an unspecific cadmium-induced increase in the passive permeability of the mitochondrial inner membrane to protons (cadmium-induced $\mathrm{H}^{+}$leak) as demonstrated in potato tuber-mitochondria using the top-down elasticity analysis [28]. The fact that the $\Delta \Psi$ imposed by succinate oxidation is not sustained and dramatically collapses, particularly for high concentrations of cadmium, can be explained due to the uncoupling action, while low cadmium concentrations inhibit solely the respiratory chain.

To our knowledge, the effects of 3,4-DCA on mitochondrial metabolism have not been characterised yet. The fluctuations on the $\Delta \Psi$ developed by mitochondria energised with succinate and those associated to the phosphorylative cycle show that 3,4-DCA completely inhibited the depolarisation induced by ADP, this means that no more phosphorylation occurs.

The effect of 3,4-DCA may be ascribed mainly to an inhibitory action on the mitochondrial respiratory chain or to some uncoupling action, or both combined.

The enhancement of the membrane potential imposed by succinate (i.e. state 4) promoted by TBT is in accordance with the effects of specific inhibitors of the ATP-synthase, such as oligomycin. In such 
is the possible contamination of the electrodes by lipophilic substances. In such cases, the utilisation of old mitochondrial preparation, for a couple of minutes, to "clean" the electrode is recommended.

Table I - Summary of quantitative effect of different substances and domestic effluents on the mitochondrial $\Delta \Psi$ in rats and in juveniles of Daphnia magna.

\begin{tabular}{|c|c|c|c|c|c|}
\hline Substance & End-Point & $\mathrm{EC}_{50}$ & $\begin{array}{c}\text { Confidence } \\
\text { Interval } \\
(95 \%) \\
\end{array}$ & $\begin{array}{c}L_{50} \\
\text { Rat } \\
\text { (mg/kg bw) }\end{array}$ & $\begin{array}{c}\mathrm{LC}_{50} \\
\text { D. magna }\end{array}$ \\
\hline Parathion & Succinate polarisation & $1.96 \mathrm{mM}$ & $0.98-3.24$ & $2.0[35]$ & $7.55 \mathrm{nM}[36]$ \\
\hline 3,4-DCA & Succinate polarisation & $\begin{array}{c}2.02 \mathrm{mM} \\
1.87 \\
1.95\end{array}$ & $\begin{array}{l}0.69-4.04 \\
1.65-2.19 \\
1.60-2.30\end{array}$ & $570.0[35]$ & $0.86 \mu \mathrm{M}[36]$ \\
\hline Endosulfan & Succinate polarisation & $\begin{array}{c}24.66 \mu \mathrm{M} \\
29.0 \\
26.40\end{array}$ & $\begin{array}{l}20.36-29.38 \\
19.00-41.21 \\
25.10-27.71\end{array}$ & $18.0[37]$ & $1.81 \mu \mathrm{M}[38]$ \\
\hline Influent & ADP depolarisation & $\begin{array}{c}2.53 \% \\
4.77 \\
5.18\end{array}$ & $\begin{array}{l}1.57-3.72 \\
3.84-5.81 \\
4.26-6.18\end{array}$ & & \\
\hline Primary Clarifier & ADP depolarisation & $\begin{array}{l}\text { N/A. } \\
\text { N/A. } \\
\text { N/A. }\end{array}$ & & & \\
\hline Clarifier & & $\begin{array}{l}\text { N/A. } \\
\text { N/A. } \\
\text { N/A. }\end{array}$ & & & \\
\hline Effluent & ADP depolarisation & $\begin{array}{l}\text { N/A. } \\
\text { N/A. } \\
\text { N/A. }\end{array}$ & & & \\
\hline TBT & ADP depolarisation & $\begin{array}{c}0.28 \mu \mathrm{M} \\
0.28 \\
0.30 \\
0.25\end{array}$ & $\begin{array}{l}0.17-0.42 \\
0.26-0.31 \\
0.27-0.34 \\
0.20-0.32\end{array}$ & $50.0-380[39]$ & $0.030 \mu \mathrm{M}[31]$ \\
\hline Cadmium & Succinate polarisation & $3.82 \mu \mathrm{M}$ & $2.80-5.00$ & $2,330[35]$ & $0.087 \mu \mathrm{M}[40]$ \\
\hline
\end{tabular}

$\mathrm{N} / \mathrm{A}=$ not applicable, hence $\mathrm{EC}_{50}$ could not be calculated due to no differences between controls and samples.

Results were reproducible for the substances tested (Table I). Changes in the mitochondrial $\Delta \Psi$ can be directly linked to changes in the external medium, caused by the presence of toxicants. The response of mitochondria to a given exposure of a chemical may affect their membrane integrity (non-specific effect) and/or specific functions (i.e. inhibition of electron transport and inhibition of ADP phosphorylation). These effects can be then quantified by the proportional potential changes to increased concentrations of xenobiotics, which can generate reliable toxicity indices with a certain degree of confidence. 
The main purpose of this study was to evaluate the suitability of an in vitro test system using isolated rat liver mitochondria for assessing the toxic effect of some chemical and effluents' fractions. It has been effectively demonstrated that the results found here qualitatively reflect the known mechanisms of mitochondrial metabolism.

Considering the numerous biochemical reactions that occur in mitochondria, when performing these tests, it is necessary to establish through screening experimentation the most appropriate endpoint.

\section{Conclusions}

Although in vitro mitochondrial activity is different from in vivo in some aspects, the mitochondrial $\Delta \Psi$ can be used in ecotoxicological research, as its experimental design allow scientifically sounding results, that can be statistically interpreted and represent an ecologically relevant endpoint, as ATP production has a meaningful importance in survival. Additionally, the $\Delta \Psi$ showed to be effective in estimating toxicity even in low concentrations and reliable EC50 could be calculated using different endpoints. The results were reproducible between tests and this also confirm the adequacy of the method in the screening of toxicity of pure substances and in toxicity identification evaluation (TIE) and toxicity reduction evaluation (TRE).

The tested substances and wastewater affected the energisation and/or the depolarisation of isolated mitochondria, and these represent physiological disruptions to the cell that can be monitored by the technique employed here.

In spite of being less sensitive than whole organism test (i.e. $D$. magna acute test), the $\Delta \Psi$ offers an additional explanation of the mode of action of a toxicant.

Mitochondria are very complex in their structure and the mode of action of distinct toxicants upon mitochondrial metabolism varies considerably and, their use in ecotoxicological testing permits the use of numerous endpoints. Therefore, the choice of the most appropriate endpoint must be defined during a preliminary test phase.

\section{Acknowledgements}

E.M. da Silva is grateful to the Brazilian Research Council (CNPq) for a post-doctoral fellowship during this research. This work was supported by JNICT, DGA (Programa Especifico do Ambiente) and the funding programmes: PRAXIS XXI and FEDER. 


\section{References}

1.Stegeman et al., Molecular responses to environmental contamination: Enzyme and protein systems as indicators of chemical exposure and effect. In: Biomarkers. Biochemical, Physiological, and Histological Markers of Anthropogenic Stress (Edited by C.H. Ward, B.T. Walton and T.W. LaPoint), Chapter 6, Lewis Pub. Boca Raton (1992).

2. L.A. Bramble, G.D. Boardman, D.R. Bevan and A.M. Dietrich, Rat liver mitochondrial and microsomal tests for the assessment of quinone toxicity, Environ. Toxicon. Chem. 13, 307-316 (1994).

3. R.W. Eastabrook and M.E. Pullman (Editors), Methods in Enzimology, Vol. 10. pp. 48-60. Academic Press, N.Y. (1967).

4. R.D. Shannon, G.D. Boardman and A.M. Dietrich, Mitochondrial response to chlorophenols as a shortterm toxicity assay, Environ. Toxicon. Chem. 10, 57-66 (1991).

5. R.K. Dubey, M.U. Beg and J. Singh, Effects of endosulfan on rat liver mitochondrial respiration and enzyme activities in vitro, Biochemical Pharmacology 33, 3405-3410 (1984).

6. R. Mishra and S.P. Shukla, Effects of endosulfan on bioenergetic properties of liver mitochondria from the freshwater catfish Clarias batrachus, Pesticide Biochemistry and Physiology 50, 240-246 (1994).

7. F.S. Darzé, Efeito do Cádmio no Crescimento e nos Metabolismos Clorofiliano e Respiratório em Lemna minor L., M.Sc. Dissertation, Federal University of Bahia, Salvador, Bahia, Brazil (1996).

8. M.E. Haubenstricker, P.G. Meier, K.H. Mancy and M.J. Brabec, Rapid toxicity testing based on yeast respiratory activity, Bull. Environ. Contam. Toxicol. 44, 669-674 (1990).

9. A.J.M. Moreno and V.M.C. Madeira, Mitochondrial bioenergetics as affected by DDT, Biochim. Biophys. Acta 1060, 166-174 (1991).

10. C.M. Palmeira, A.J. Moreno and V.M.C. Madeira, Interactions of herbicides 2,4-D and dinoseb with liver mitochondrial bioenergetics, Toxicology and Applied Pharmacology 127, $50-57$ (1994).

11. C.M. Palmeira, A.J. Moreno and V.M.C. Madeira, Mitochondrial bioenergetic is affected by the herbicide paraquat, Biochimica et Biophysica Acta 1229, 187-192 (1995). 
12. M. Bragadin and P. Dell'Antone, Mitochondrial bioenergetics as affected by cationic detergents, Arch. Environ. Contam. Toxicol. 30, 280-284 (1996).

13. A.J.M. Moreno, Estudo do Efeito de Insecticidas de Uso Comum na Bioenergética Mitocondrial. Ph.D. Thesis, University of Coimbra, Coimbra, Portugal (1992).

14. A.G. Gournall, C.J. Bardawill and M.M. David, Determination of serum proteins by means of the biuret, J. Biol. Chem. 177, 751-766 (1949).

15. N. Kamo, M. Muratsugu, R. Hongoh and V. Kobatake, Membrane potential of mitochondria measured with an electrode sensitive to tetraphenyl phosphonium and relationship between proton electrochemical potential and phosphorylation potential in steady state, J. Membr. Biol. 49, 105-121 (1979).

16. B.D. Jensen and T.E. Gunther, The use of tetraphenylphosphonium (TPP+) to measure membrane potentials in mitochondria: membrane binding and respiratory effects, Biophys. J. 49, 105-121 (1984).

17. D.E. Wingrove and T.E. Gunther, The Na dependent $\mathrm{Ca}$ efflux in rat liver mitochondria, Fed. Proc. Fed. Am. Soc. Exp. Biol. 44: 1082-1087 (1985).

18. A. Masini, D. Ceccarelli-Stanzani and U. Muscatello, An investigation on the effect of oligomycin on state-4 respiration in isolated rat-liver mitochondria, Biochim. Biophys. Acta 767, 130-137 (1984).

19. H. Zar, Biostatistical Analysis ( $2^{\text {nd }}$ Edn), p. 662. Prentice-Hall, London (1994).

20. P. Mitchell, Chemiosmotic coupling and photosynthetic phosphorylation, Biol. Rev. 41, 445-501 (1966).

21. D.G. Nicholls, Bioenergetics. Introduction to the Chemiosmotic Theory. Acad. Press, N.Y. (1982).

22. I. Simões and P. Castanheira, Efeito de pesticidas de uso comum em parâmetros bioenergéticos mitocondriais. Internal Report. University of Coimbra, Coimbra, Portugal (1997).

23. M.D. Brand, The proton leak across the mitochondrial inner membrane, Biochim. Biophys. Acta 1018, 128-133 (1990). 
24. T. Yamano and S. Morita, Effects of pesticides on isolated rat hepatocytes, mitochondria, and microsomes. Arch. Environ. Contam. Toxicol. 25, 271-278 (1993).

25. A. Wenzel, M. Nendza, P. Hartmann and R. Kanne, Testbattery for the assessment of aquatic toxicity, Chemosphere 34, 307-322 (1997).

26. S. Miccadei and A. Floridi, Sites of inhibition of mitochondrial electron transport by cadmium, Chemico-Biological Interactions 89, 159-167 (1993).

27. E.M. Diamond and J.E. Kench, Effects of cadmium on the respiaratory of rat liver mitochondria, Environ. Physiol. Biochem., 4, 280-305 (1974).

28. A. Kesseler and M.D. Brand, Localization of the sites of action of cadmium on oxidative phosphorylation in potato tuber mitochondria using top-down elasticity analysis. Eur. J. Biochem., 225, 857-906 (1994).

29. W.N. Aldridge and B.W. Street, Oxidative phosphorylation, biochemical effects and properties of trialkytins, Biochem. J. 91, 287-297 (1964).

30. M. Muller, L. Renberg, G. Rippen and A. Bjorseth, Tributyltin in the environment. Sources, fate and determination. Water. Water Pollution Research Report 8, EUR 11562 (1988).

31. P. Miana, S. Scotto, G. Perin and E. Argese, Sensitivity of Selenastrum capricornutum, Daphnia magna and submitochondrial particles to tributyltin, Environmental Technology 14, 175-181 (1993).

32. D.J. Baird, I. Barber, M. Bradley, P. Calow, and A.M.V.M. Soares, The Daphnia bioassay: a critique, Hidrobiology 188/189, 403-406 (1989).

33. A.M.V.M. Soares, D.J. Baird and P. Calow, Interclonal variation in the performance of Daphnia magna Straus in chronic bioassays. Environ. Toxicol. Chem. 11, 1477-1483 (1992).

34. R.H. Rimpau, Toxicity of endosulfan and its metabolites. In: Residue Reviews. Properties, Effects, Residue, and Analytics of the Insecticide Endosulfan (Edited by H. Goebel, S. Gorbach, W. Knauf, R.H. Rimpau and H. Hüttenbach), Chapter 4, Springer-Verlag, Berlin (1982).

35. IUCLID, International Uniform Chemical Information Database, Version 1.0 (1996). 
36. L. Guilhermino, M.C. Lopes, A.P. Carvalho and A.M.V.M. Soares, Ihnibition of acetylcholinesterase activity as effect criterion in acute tests with juvenile Daphnia magna, Chemosphere 32, 727-738 (1996).

37. ARSIM, Agricultural Research Service, USDA, Information Memorandum, Beltsville, MD, USA, 20, (1966).

38. A.E. Lemke, Comprehensive Report. Interlaboratory comparison of acute testing set. U.S. EPA Environmental Research Laboratory, Duluth, Minnesota. In Ambient Water Quality Criteria for Endosulfan. U.S. EPA, Washington, DC, USA (1980).

39. R. Eisler, Tin hazards to fish, wildlife, and invertebrates: A synoptic review. US Department of the Interior Fish and Wildlife Service, Patuxent Wildlife Research Center,. Contaminant Hazard Reviews Report No 15, Laurel, MD, USA (1989).

40. L. Guilhermino, T.C. Diamantino, R. Ribeiro, F. Gonçalves and A.M.V.M. Soares, Suitability of test media containing EDTA for the evaluation of acute metal toxicity to Daphnia magna Straus, Ecotoxicology and Environmental Safety (in press). 